\title{
Mädchen schnuppern in technische Berufe
}

Zum ersten Mal fand in der Siemens-Niederlassung Innsbruck auf Initiative der Volkswirtschaftlichen Gesellschaft Tirol und dem JUFF Frauenund Jugendreferat der "Girls' Day" statt. Dabei besuchten Mädchen der achten und neunten Schulstufe einen Tag die Siemens-Lehrwerkstatt, um die technischen Berufe eines Elektronikers, Elektro-Betriebstechnikers und EDVTechnikers kennen zu lernen. Unter fachkundiger Anleitung führten die Mädchen Löt- und Bohrübungen durch und fertigten manuell eine elektroni- sche Schaltung. Danach wurde eine solche Schaltung zusätzlich am PC programmiert.

\section{Schnupperlehre}

Seit 1989 bietet Siemens in Innsbruck die „Schnupperlehre" an. Schüler und Schülerinnen der neunten Schulstufe können eine Woche lang alle drei Berufe - Elektroniker, Elektro-Betriebstechniker und EDV-Techniker - kennen lernen. Seit rund vier Jahren sind etwa $15 \%$ der Teilnehmer weiblich. Den Abschluss bildet ein Beurteilungs- und Beratungsgespräch über den
Wunschberuf und die erbrachte Leistung. In den letzten zehn Jahren wurden jährlich ein bis zwei Schnupperlehrlinge in die Ausbildung übernommen. Derzeit bildet die Niederlassung Innsbruck 16 gewerbliche und drei kaufmännische Lehrlinge aus wurde mit der Tiroler Landesauszeichnung „Ausgezeichneter Lehrbetrieb" bedacht.

Informationen: Siemens AG Österreich, Sylvia Schwarz, Siemensstraße 88-92, A-1210 Wien, Telefon +43 (5) 1707 24045, E-Mall: sylvia.schwarz@siemens.com, Internet: www.siemens.at

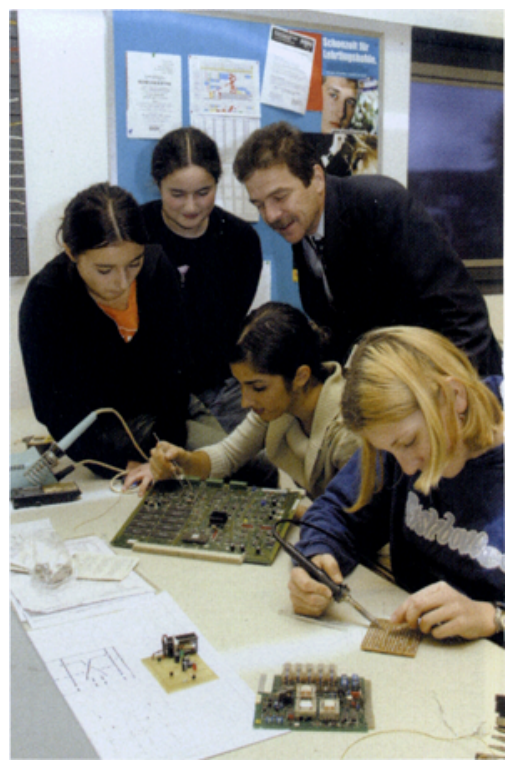

Am Girls' Day besuchten

Mädchen der achten und neunten Schulstufe (im Bild mit Niederlassungsleiter Dipl.-Ing. Walter Mayr) die Lehrwerkstatt der SiemensNiederlassung Innsbruck

\section{SESAMES-PREIS}

\section{Chips von Infineon und Sony ausgezeichnet}

Anlässlich der Messe „Cartes 2002“, die Anfang November in Paris stattfand, wurden die neuen Mikrocontroller für kontaktlose Chipkartenanwendungen von Sony und Infineon Technologies mit dem Sesames-Preis für die beste technologische Innovation im Jahr 2002 ausgezeichnet. Eine internationale Jury von neun Branchenkennern prämierte herausragende Leistungen in der Chipkartenbranche.

Infineon und Sony erhielten den Technologie-Innovationspreis für ihren gemeinsam entwickelten Mikrocontroller für kontaktlose Chipkartenanwendungen, den SLE66CLX320PS, und Infineon für seinen eigenen Sicherheitscontroller, den

SLE66CLX320P. Die prämierten Chipkartencontroller unterstützen die drei derzeit marktbeherrschenden kontaktlosen Schnittstellenformate ISO/IEC 14443 Typ A, Typ $B$ und Sonys Felica®. Diese unterscheiden sich bezüglich der Protokolle für die Datenübertragung.

Kontaktlose Chipkartenanwendungen

Im Gegensatz zu kontaktbe-

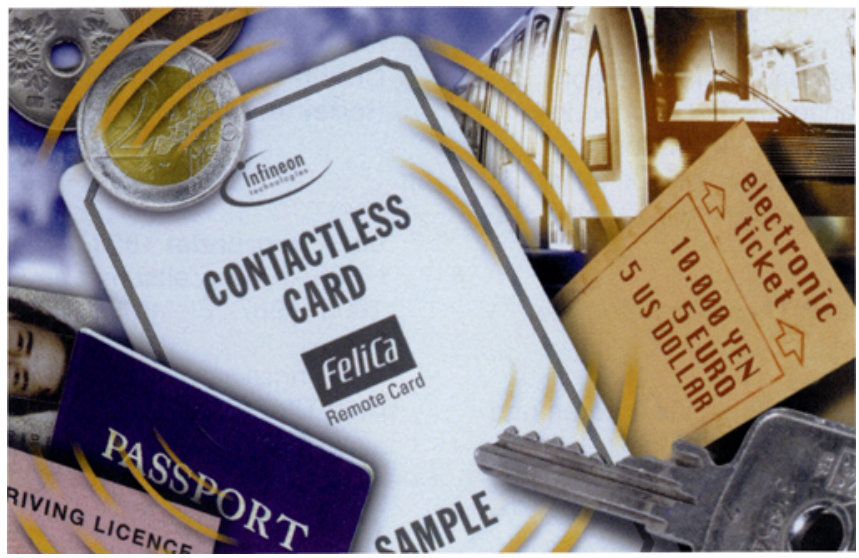

hafteten Chipkarten, bei denen die Daten zwischen Chipkarte und Lesegerät über Kontakte ausgetauscht werden, muss man die so genannten kon- taktlosen oder Dual-InterfaceChipkarten zur Datenübertragung nicht in ein Lesegeräte einschieben. Sie kommunizieren per Funk mit dem Terminal.
Die prämierten Chips bieten Authentifizierung und Identifikation mit schnellem Datenaustausch, verfügen über Verschlüsselungs- und Entschlüsselungsfunktionen und ermöglichen sichere und zuverlässige Transaktionen. Mit innen bestückte Chipkarten können vielseitig als elektronische Tickets, Firmenoder Behördenausweise sowie als Bankenkarten eingesetzt werden.

Informationen: Infineon Technologies, Microelectronıc Desıgn Centers Austria GmbH, Development Center Villach, Edith Prugger, Siemensstraße 2, A-9500 Villach, Telefon +43 (4242) 305-6341, E-Maıl: edith.prugger@infineon.com, Internet: www.infineon.com

\section{AKTUELLE STUDIE \\ Mobilfunktrends in Österreich}

Im September 2002 führte mobilkom austria gemeinsam mit dem Marktforschungsinstitut Fessel GfK eine Studie durch, die den Einfluss des Handys auf das Leben der Österreicherinnen und Österreicher untersuchte. Bei jungen Usern bis 24 Jahre ist die Prozentzahl der Handyuser am höchsten: In dieser Altersgruppe haben $91 \%$ das Handy immer dabei, gesamt betrachtet $79 \%$ aller ÖstereicherInnen.

\section{Erreichbarkeit und Handy- Freezones}

Für mehr als Dreiviertel der befragten Personen ist es wichtig, für Freunde ständig erreichbar zu sein. Das hat zur Folge, dass über die Hälfte der User (51 \%) das Handy gar nicht mehr ausschalten. Der Trend geht in die Richtung, das Handy, wenn nötig, auf lautlos zu stellen, aber 